\title{
New PET system permits reliable estimates of myocardial blood flow and flow reserve
}

\author{
Nagara Tamaki, MD, PhD ${ }^{\mathrm{a}}$ \\ a Department of Nuclear Medicine, Hokkaido University Graduate School of Medicine, Sapporo, \\ Japan
}

Received Feb 9, 2015; accepted Feb 9, 2015

doi: 10.1007/s12350-015-0098-3

See related article, pp. 457-472

A significant progress in positron emission tomography (PET) system has recently been seen which may improve sensitivity, spatial resolution, and noise reduction. Such progress has provided better quality of myocardial perfusion images with lower injected dose, and thus lower radiation to the patient. In addition, better quantification of myocardial blood flow (MBF) and coronary flow reserve (CFR) is expected. ${ }^{1}-^{7}$

Many of new PET cameras have three-dimensional (3D) mode acquisition with time-of-flight (TOF) technology. The system sensitivity in 3D acquisition is much greater than $2 \mathrm{D}$ acquisition. On the other hand, the fraction of background activity is also higher in $3 \mathrm{D} .^{8}$ TOF, on the other hand, may significantly increase signal-to-noise ratio by decreasing noise. ${ }^{8}$ TOF can estimate the arrival time of the $511 \mathrm{keV}$ photons, and therefore, the location of the emission point may be constrained along a line-of-response between the two detector pairs. ${ }^{9,10}$ In order to perform adequate effects of $\mathrm{TOF}$, a scintillation detector with high time resolution is required. The main factor that permits recent TOF PET development is the availability of new scintillation materials, such as LSO and LYSO. In addition, TOF PET has gained greater advantages in whole-body imaging with wide field of view than the brain imaging. A number of reports indicated clinical value of TOF PET in oncology fields. On the other hand, limited number of

Reprint requests: Nagara Tamaki, MD, PhD, Department of Nuclear Medicine, Hokkaido University Graduate School of Medicine, N-15, W-7, Kita ku, Sapporo 060-8638, Japan; natamaki@med.hokudai. ac.jp

J Nucl Cardiol 2016;23:473-4.

$1071-3581 / \$ 34.00$

Copyright (C) 2015 American Society of Nuclear Cardiology. reports have reported clinical value of TOF PET in cardiac imaging. ${ }^{11,12}$ Cardiac PET may require reliable quantification of serial tracer concentrations, and thus, it is often used to estimate MBF, CFR, and various metabolic and molecular functions as quantitative parameters, such as metabolic rate of glucose, transporter retention index, receptor density, etc. Such cardiac functional assessment using optimal PET parameters is expected to be applied for severity assessment, treatment planning, and treatment monitoring in the near future.

Masaya et al in the current issue assessed reproducibility of MBF and CFR with N-13 ammonia PET under 3D acquisition with and TOF. ${ }^{13}$ The phantom study showed smaller partial volume effect with higher image contrast with TOF acquisition than non TOF. Such improvement was better seen in larger field of view than smaller field of view. Such results are quite reasonable on the basis of TOF concept. In addition, clinical data also showed higher reproducibility with smaller intra- as well as inter-observer variability using TOF technique. Unfortunately, this reproducibility study was not designed based on two separate acquisition protocols. However, small intra- as well as inter-observer variability of MBF and CFR values was well demonstrated, probably due to higher target-to-background ratio and less partial volume effects. Interestingly, the authors pointed out possible differences in variation in the apex and the segments near the right ventricle with and without TOF acquisitions. Higher background noise from the right ventricle may cause variations without TOF. On the other hand, there are not clear explanations why TOF may reduce variation of MBF value in the apex. Partial volume effects may significantly be improved by TOF, particularly in smaller thickness and also peripheral field of view for apical areas.

There are a number of issues which should be solved in the future. The current study was done with two separate reconstruction methods in each study 
(TOF-OSEM and 3D-RAMLA). A different reconstruction software might possibly create somewhat different results. In addition, N-13 ammonia is not commonly used PET perfusion tracer since this may require in-house cyclotron, despite this tracer has been approved for clinical use in the USA and Japan. Similar analysis should be done for rubidium-82 which emits higher energy and higher counts with more injected dose to a patient. ${ }^{14}$ Since 3D TOF PET camera has recently become a state-of-the-art PET system, many more patient data will be collected and analyzed with this system within a few years. A new outcome analysis and correlative study with various clinical risk factors should be performed with many patient data base.

\section{References}

1. Saraste A, Kajander S, Han C, Nesterox S, Knuuti J. PET: IS myocardial flow quantification a clinical reality? J Nucl Cardiol. 2012;19:1044-59.

2. Schindler TH, Quercioli A, Valenta I, Ambrosio G, Wahl R, Disizian V. Quantitative assessment of myocardial blood flow. Clinical and research applications. Semin Nucl Med. 2014;44:274-93.

3. Naya M, Tamaki N, Tsutsui $\mathrm{H}$. Coronary flow reserve estimated by positron emission tomography to diagnose significant coronary artery disease and predict cardiac events. Circ J. 2015;79:15-23.

4. Camici P, Rimoldi OE. The clinical value of myocardial blood flow measurement. J Nucl Med. 2009;50:1076-87.
5. Bengel FM, Higuchi T, Javadi M, Lautamaki R. Cardiac positron emission tomography. JACC. 2009;54:1-15.

6. Schelbert HR. Anatomy and physiology of coronary blood flow. J Nucl Cardiol. 2010;17:545-54.

7. Yoshinaga K, Tomiyama Y, Suzuki E, Tamaki N. Myocardial blood flow quantification using positron emission tomography. Circ J. 2013;77:1662-71.

8. Turkington TG. PET physics and PET instrumentation. In: Wahl RL, editor. Principles and Practice of PET and PET/CT. 2nd ed. Wolters: Kluwer; 2009. p. 47-57.

9. Ter-Pogossian MM, Ficke DC, Yamamoto M, Hood JT, Super PETTI. Super PETTI: A positron emission tomograph utilizing photon time-of-flight information. IEEE Trans Med Imaging. 1982;1:179-87.

10. Pend $\mathrm{H}$, Levin CS. Recent developments in PET instrumentation. Curr Pharm Biotechnol. 2010;11:555-71.

11. Slomka PJ, Berman DS, Germano G. New cardiac cameras: single-photon emission CT and PET. Semin Nucl Med. 2014;44:23251.

12. Schaefferkoetter J, Ouyang J, Rakvongthai Y, Nappi C, El Fakhri G. Effect of time-of-flight and point spread function modeling on detectability of myocardial defects in PET. Med Phys. 2014; 41(6):062502.

13. Masaya S, Onoguchi M, Tomiyama T, Ishihara K, Takahashi N, Sakurai M, Matsumoto K, Kumita K. The reproducibility of timeof-flight PET and conventional PET for quantification of myocardial blood flow and coronary flow reserve with ${ }^{13} \mathrm{~N}$-ammonia. (Current paper)

14. Klein R, Beanlands RSB, deKemp RA. Quantification of myocardial blood flow and flow reserve. J Nucl Cardiol. 2010;17: $555-70$ 